\title{
Life After AREDS 2: What Should We Recommend to Patients With or at Risk of AMD?
}

\author{
Langis Michaud ${ }^{\mathrm{A}}$ \\ Julie Brûlé ${ }^{B}$ \\ Jean-Sebastien Dufour ${ }^{\mathrm{C}}$ \\ Pierre Forcier ${ }^{\mathrm{D}}$ \\ Guillaume Fortin $^{\mathrm{E}}$ \\ Kevin Messier ${ }^{\mathrm{F}}$ \\ Marc-André Rhéaume ${ }^{\mathrm{G}}$ \\ Yvon Rhéaume ${ }^{\mathrm{C}}$ \\ Patrick Simard ${ }^{\mathrm{c}}$

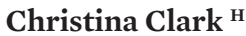

\section{A : Professor, Université de Montréal - coordinator of this group}

Participants :

B : Adjunct Professor, Université de Montréal

C : Clinical Instructor, Université de Montréal

D : Associate Professor, Université de Montréal

E : Private Practitioner

$\mathrm{F}$ : Optometrist- residency in ocular health- Institut de l'Oeil

des Laurentides (OD-MD center)

G: M.D. Ophthalmologist

H: Medical writer

\begin{abstract}
PURPOSE

To establish a consensus on clinical recommendation of oral supplementation for patients with or at risk of developing age-related macular degeneration (AMD), from the perspective of the Age-Related Eye Disease Study 2 (AREDS 2) and other studies.
\end{abstract}

\section{METHODS}

Panel discussion based on a literature review of pertinent articles related to the prevention of AMD with oral supplementation.

\section{RESULTS}

On the basis of the findings, patients must first be encouraged to modify their diet and to eliminate modifiable risk factors before being recommended any type of oral supplementation. Then, recommendations must be customized on the basis of a patient's individual risk profile (i.e., age, gender, heredity, etc.) and severity of disease (i.e., category 1 to 4 ). Essential fatty acids (omega-3s) and vitamins may play a role, in a given clinical population, to prevent the occurrence or the progression of AMD disease. However, there is no single formula that can be applied to all patients with or at risk of AMD.

\section{CONCLUSIONS}

This group concluded that the full body of literature must be taken into consideration in order to justify clinical recommendations for patients. A single study such as AREDS 2 cannot, by itself, guide clinical practice. In all cases, recommendations must be individualized and patients should be monitored regularly.

KEY WORDS:

age-related macular degeneration, poly-unsaturated fatty acids, vitamins, AREDS 2 
Sommaire

BUT

Établir des recommandations cliniques consensuelles quant à la gestion clinique des patients atteints ou à risque de développer une dégénérescence maculaire liée à l'âge (DMLA).

\section{MÉTHODES}

Discussion d'un panel d'experts basée sur l'analyse de divers articles scientifiques relatifs à la prise de suppléments vitaminiques et nutritionnels chez des patients atteints ou à risque de DMLA.

\section{RÉSULTATS}

Selon le panel, suite à l'analyse des articles, la première intervention devrait être d'inciter le patient à améliorer son hygiène de vie avant de recourir à des suppléments oraux.

Par la suite, les recommandations cliniques doivent tenir compte du profil de risque du patient, de sa nutrition, de sa condition systémique ainsi que de l'état de sa santé oculaire. Les omégas 3s et les vitamines peuvent jouer un rôle bénéfique auprès de populations cibles afin de prévenir l'apparition ou l'évolution de la DMLA. Comme il n'existe pas de recettes uniques, le tout doit être personnalisé selon les besoins du patient.

\section{CONCLUSION}

Le groupe conclut que l'ensemble de la littérature doit être prise en compte afin de justifier le recours à des suppléments oraux (omégas et vitamines) et que les recommandations doivent être personnalisées. Une seule étude, comme AREDS 2, bien que très importante, ne peut déterminer à elle-seule le comportement clinique des professionnels de la vue. L'importance du suivi régulier du patient doit également être comprise par tous.

A ge-related macular degeneration (AMD) is the leading cause of irreversible vision loss in developed countries. ${ }^{1,2}$ It is estimated that there are 17,000 new cases of neovascular (NV) AMD and 180,000 new cases of geographic atrophy (GA) AMD in Canada every year. $^{3}$ The disease has a substantial negative impact on patient quality of life and imposes a considerable burden on the economy. ${ }^{3}$ Early and intermediate stages of AMD are prevalent in people older than 65 years of age, ${ }^{4}$ and without intervention, the condition can evolve to advanced $\mathrm{AMD}^{5}$ and result in significant loss of visual function.

Published 12 years ago, the Age-Related Eye Disease Study (AREDS) demonstrated that in persons with intermediate to severe AMD, a daily oral supplement containing vitamins and antioxidants reduced the risk of progression to advanced AMD by $25 \%$ versus placebo over a period of 5 years. ${ }^{6}$ This "AREDS formula" consisted of 500 milligrams (mg) vitamin C, 400 international units (IU) of vitamin $\mathrm{E}, 15 \mathrm{mg}$ beta-carotene, $80 \mathrm{mg}$ zinc oxide, and $2 \mathrm{mg}$ cupric oxide. Since then, observational studies have suggested that dietary intake of other carotenoids, particularly lutein and zeaxanthin, might play a role in protecting against AMD. ${ }^{78}$ Moreover, the authors of AREDS ${ }^{9,10}$ and others ${ }^{8,11-13}$ highlighted the important role of dietary or supplemental forms of omega-3s, for preventing the development of AMD or its progression.

This is the context in which the AREDS 2 was published in May 2013.14 This study, which was initiated in 2006, demonstrated that the addition of lutein, zeaxanthin, and omega-3s to the original AREDS formula, did not further reduce the risk of progression to advanced AMD 
relative to the original formula. ${ }^{14}$ However, several secondary and subgroup analyses in this study suggested a benefit to replacing beta-carotene, which is associated with an increased risk of lung cancer in current and former smokers, with other carotenoids such as lutein and zeaxanthin. These conflicting results, notably with regard to omega-3s, have led to confusion among health care professionals about how to counsel patients and their caregivers about dietary strategies to prevent the development and progression of AMD.

On November 1, 2013, a group of Quebec experts, consisting of eight optometrists and one ophthalmologist, gathered in Montreal to discuss the outcomes of the AREDS 2 from a clinical perspective, with the goal of guiding the optimal management of this disease. In order to consider the AREDS 2 in a more global context, a general critique was undertaken. Then, each participant was assigned to review and present the key findings of a published article related to the use of dietary supplements or dietary factors associated with AMD (see Table $\mathbf{1}$ for a brief synopsis of the studies and key findings). A facilitated group discussion that ensued focused on synthesizing the data with the eventual goal of developing a clear and practical set of consensusbased, nonbinding clinical recommendations for patients with AMD.

\section{GENERAL CRITIQUE OF THE AREDS 2}

First, it is important to recognize that the AREDS 2 was founded on the original AREDS. A socioeconomic analysis of the patients enrolled in this latter study showed that they were, on average, more educated and better nourished at baseline than the average American ${ }^{14}$ as well as those attending optometry practices in Canada. Moreover, a substantial proportion of patients were already taking vitamin and antioxidant supplements, ${ }^{6,14}$ which suggested that their overall nutrition status was already supported by an external source of these nutrients. In this regard, there was no real placebo group in the AREDS 2 . Moreover, $14 \%$ of patients were additionally taking "nonauthorized" supplements, which further increased their antioxidant intake. ${ }^{14}$ Furthermore, the dosage and formulation of omega-3 supplements that were used in this study (eicosapentaenoic acid greater than docosahexaenoic acid [EPA $>$ DHA] $1000 \mathrm{mg} /$ day versus $2000 \mathrm{mg} /$ day, as esters or triglycerides) were not optimal, considering the results of earlier studies on this subject. Finally, the AREDS 2 evaluated progression of AMD from moderate to advanced disease ( $\mathrm{NV}$ and GA forms) without considering the effects of supplementation on the risk of development of the disease or its progression from mild to moderate disease.

The conclusions of the AREDS 2 can be applied to patients who were similar to those who were evaluated in the study, that is, patients with moderate to advanced AMD who are well nourished and well educated. Before extrapolating the study results to other patients, evidence derived from other studies must first be considered in order to appreciate the general context from which clinical recommendations can be formulated.

\section{GENERAL RECOMMENDATIONS}

After considering the outcomes of several studies that were presented and keeping a general context in mind, a consensus was reached by this group of experts-that recommendations should be based on the individual patient and their particular risk profile. Health care professionals should assess a patient's modifiable risk factors at the earliest opportunity to better counsel and categorize their risk of developing AMD or its progression to advanced AMD and to tailor advice about lifestyle, diet, and supplements. Tools are available to assist health care professionals in this regard. The results of the Macular Assessment Program (MAP), which aimed at evaluating the perceptions versus the realities of 290 Canadian optometrists, were recently reported and reviewed at this meeting. ${ }^{15}$ This tool enables optometrists to categorize patients as having low, moderate, or high risk of AMD, on the basis of an evaluation of their modifiable and nonmodifiable risk factors. The literature suggests that the most important modifiable risk factors are smoking, alcohol consumption ( $>3$ standard drinks/day), sun exposure (photostress), poor-quality diet, obesity, cardiovascular risk factors, and adherence to medication, whereas the most important nonmodifiable risk factors include age, gender, family history, ethnicity (Caucasians), and socioeconomic and educational status.

Therefore, all patients should be strongly encouraged to act on their modifiable risk factors 
including smoking, diet, exercise, weight control, cholesterol, sun protection, and cardiovascular risk factors. This should constitute the foundation of counselling for all patients at risk of AMD or with AMD of any severity. Dietary advice should include information on food sources of lutein and zeaxanthin (e.g., green leafy vegetables and canned corn) and omega-3 fatty acids (e.g., wild fatty fish such as salmon, herring, and mackerel).

\section{RECOMMENDATIONS STEMMING FROM THE AREDS 2}

When a health care professional decides to recommend a vitamin supplement to a patient, the general consensus is that lutein and zeaxanthin should be preferred over beta-carotene. There is a growing body of evidence suggesting that high-dose beta-carotene supplementation $\left(20-30 \mathrm{mg} /\right.$ day) is associated with a higher risk of lung cancer in smokers. ${ }^{14,16}$ The AREDS 2 confirmed that any patient who actively smoked in the past must be considered a smoker, regardless of the duration since cessation. This is an important point because the prevalence of current and ex-smokers is higher in Quebec compared with the national average. According to the Canadian Tobacco Use Monitoring Survey, in 2012, 14.9\% of the Quebec population aged 45 years and older identified themselves as current smokers and a further $44.7 \%$ were former smokers. ${ }^{17}$

\section{RECOMMENDATIONS BASED ON CATEGORY OF AMD}

\section{Primary Prevention of AMD}

Among the group of experts of the AREDS 2, it was felt that unaffected people with risk factors for the disease (e.g., family history or genetic risk) had been less well studied in randomized controlled trials and that the potential benefits of supplementation with antioxidants remains unclear for them. However, strong evidence from observational studies suggests that diets rich in omega-3 fats and fish intake are associated with protection against the development of AMD. ${ }^{8,9,12,18}$ Consequently, eye health professionals may consider recommending omega-3 supplements or a diet rich in omega-3 sources in patients with risk factors for AMD (e.g., genetics), especially those who are poorly nourished, rather than recommending antioxidant supplements. Most of the experts also agreed that the safety and tolerability profile for omega-3 supplements was favourable and that supplementation did not appear to introduce unacceptable risks. There was stronger consensus among this group of experts that with regard to other modifiable risk factors, counselling that includes recommendation of regular exercise, sun protection, weight control, control of cardiovascular risk factors, and cessation of smoking, is very important and should not be neglected as a first step to prevent AMD occurrence.

\section{Progression from Early AMD to Advanced AMD}

The AREDS failed to demonstrate any significant benefit of supplementation in patients with less severe AMD (i.e., Category 1 or 2 ). ${ }^{6}$ In this study, very few patients with Category 2 disease at baseline (i.e., patients with extensive small drusen, pigment abnormalities, or a few intermediate drusen) developed advanced AMD over the study period. On the basis of the available data, some clinicians therefore would not explicitly recommend antioxidant supplements to individuals with early AMD unless there was clear evidence of poor diet quality or dietary intake of carotenoids and omega-3s was insufficient. Dietary interventions may be of value in reducing the risk of progression in these patients.

\section{Intermediate or Advanced AMD}

For those patients who already have advanced AMD (i.e., defined by AREDS category), there may be some benefit in taking an antioxidant supplement with or without omega-3s, given that both the AREDS and the AREDS 2 showed a 25 to $30 \%$ reduction in the risk of progression from moderate to advanced AMD in the affected eye, as well as progression in the other eye.,14 Patients who are most likely to benefit from supplements are those who fit the profile of the patients studied in the AREDS and AREDS 2, that is, those with category 3 (many intermediate drusen or at least one large druse with abnormal pigmentation) or category 4 AMD (GA affecting the fovea or wet AMD with retinal fibrosis). ${ }^{14}$

Some experts still believe that patients with intermediate or advanced AMD could also benefit from omega-3 fatty acid supplementation despite the negative findings of the AREDS ${ }^{2,14}$ 
particularly older patients who often have comorbid conditions (cardiovascular disease, diabetes, etc.), in whom the beneficial effects of fatty acid supplementation have been more clearly demonstrated. However, at this time, given the available evidence from randomized controlled trials, the optimal dose of omega- 3 supplements, duration of treatment, and the magnitude of potential benefit remain unclear. There is consistent evidence from observational studies that people who consume the highest amount of omega-3s, particularly in the form of triglycerides or fatty fish in their diet have a lower incidence of AMD or progression to advanced AMD compared with those with the lowest intake.,12,18 Moreover, supplementing the diet with omega-3s, through either diet or supplements, could offer ancillary benefits, notably in patients presenting with symptoms of dry eyes, which is often the case in patients with AMD.

It was acknowledged that many patients with AMD want to do something to try to improve their health and prevent the progression of their disease. Taking a supplement that offers a potential $25 \%$ reduction in the risk of progression ${ }^{6}$ may offer them hope and a sense of control over their disease. Notably, evidence suggests that people with the lowest dietary intakes of antioxidants ${ }^{14}$ and those under 75 years of age ${ }^{7}$ may derive the greatest benefits from supplementation. These patients might therefore constitute the best group to target in terms of clinical recommendations around diet and supplements. This further underscores the importance of classifying patients according to their risk of advanced AMD in the clinical setting.

\section{SHOULD WE RECOMMEND SUPPLEMENTS VERSUS DIETARY INTERVENTIONS?}

This group of experts acknowledged that patients must first be counselled to modify their diet to include green vegetables (source of lutein and zeaxanthin), and carotenoids and vitamins (fruits and vegetables), as well as fatty fish, if possible from wild sources, several times a week. For example, some studies reported benefits of consuming four or more portions of fish weekly ${ }^{18}$ In addition to the quantity of fish, the type of fish may also be influential with respect to outcomes. ${ }^{12}$ Moreover, clinicians should be cognizant of the tendency of patients to overreport or overstate adherence to dietary recommendations. The populations studied tended to be better nourished and more highly educated than the general population, particularly in the AREDS ${ }^{6}$ and the AREDS $2 .{ }^{14}$ This might have biased the results of studies. Consequently, it seems logical to recommend supplements to people with nutritional deficiencies and risk factors for AMD, as well as for people living alone (who are often less well nourished) in the hope that outcomes will be superior to those expected in well-nourished patients.

Once supplementation is recommended, it is essential to assess adherence during followup visits. One should not assume that patients are always adherent to their prescribed regimen. Factors that may limit patient adherence to dietary supplementation include lack of perceived benefit (e.g., no effects on vision), cost of supplements, size of pills, frequency of dosing, and potential problems with tolerability (e.g., gastrointestinal discomfort). Eye care professionals should be prepared to discuss the reasons for recommending supplements and help their patients set reasonable goals. From the start, it should be made clear to patients that supplements do not improve vision but that they are meant to reduce the risk of progression to more advanced disease. The AREDS suggested that in patients with category 3 or 4 AMD who are generally well nourished, the risk of progression to advanced disease may be reduced by 25 to $30 \%$ with antioxidant supplementation. ${ }^{6}$

\section{INFORMATION FOR OTHER HEALTH CARE PROFESSIONALS}

Vitamin and nutritional supplements can have an impact on a patient's medication regimen. When in doubt, eye care professionals should seek advice from the pharmacist to better evaluate the risk of potential drug-nutrient interactions. Although there have been some concerns about the use of supplements in patients with existing renal dysfunction, in the clinical experience of this group of advisors, such problems have been uncommon. Patients should also be reminded to inform their family physician about any intake of vitamins or omega-3s, notably when taking other medications such as warfarin or antidiabetic drugs, since the dosage of these might need to be adjusted based on the patient's response to omega-3s. 


\section{RECOMMENDATIONS FOR OPTIMAL FREQUENCY OF FOLLOW-UP}

Timing of follow-up is an important consideration because the optometrist needs to see the intermediate AMD patient at the right time to see progression of the disease. Like recommendations for supplements and diet, the frequency of follow-up should ideally be individualized to the patient's risk profile and needs. It was generally agreed that patients with milder cases of AMD (i.e., category 2 or lower) should be seen at least annually. The majority of patients with category 3 AMD should be monitored every 6 months and, in some cases, more often. Patients with NV AMD should be seen more frequently by their ophthalmologist (i.e., three or four times annually). Self-report of symptoms using the Amsler grid does not substitute for clinic visits, although it can complement the tools used for assessing the progression of disease. Finally, clinical visits represent an opportunity for optometrists to educate patients about modifiable risk factors, the importance of following clinical recommendations, and to modify the treatment and follow-up plan as needed.

\section{EXAMINATION OF THE PATIENT WITH AMD}

In a perfect world, optical coherence tomography (OCT) would be used to test all patients with AMD to monitor for progression to neovascular disease. However, given the limited resources, judicious use is of this technology is necessary. It was suggested that a baseline OCT followed by annual testing might be appropriate in Category 3 or 4 patients with no new symptoms or complaints and without evidence of hemorrhage or exudate on clinical examination. OCT is not mandatory but is recommended in patients with dry AMD, especially if a visual acuity change occurs. Fundus photography is a good tool to document a patient's status and allow for easy evaluation of a patient's disease progression. In addition, it can be beneficial to show patients pictures of their eye examination, results of OCT, or both to help them understand their disease and its eventual progression and thereby promote greater adherence to the proposed treatment regimen.

For patients with category 1 or 2 AMD, use of the Amsler grid remains appropriate despite suboptimal sensitivity and specificity. This at-home test is particularly helpful for patients to self-evaluate the progression of their eye disease. Optometrists must remember to instruct patients to test one eye at a time.

\section{CONCLUSION}

The first step in the management of a patient with AMD is to identify the disease and determine its severity. Recommendations must be customized based on a patient's individual risk profile (i.e., age, gender, heredity, etc.) and severity of disease (i.e., category 1 to 4). There is no single formula that can be applied to all patients with or at risk of AMD.

Patients must, first and foremost, be encouraged to modify their diet and to eliminate modifiable risk factors such as smoking, sedentary lifestyle, excessive alcohol intake, and so on. The patient should be encouraged to exercise regularly. Medication adherence (for hypertension, hypercholesterolemia, diabetes, etc.) must be reinforced. Taking a supplement must not be a substitute for making healthy lifestyle changes.

Next, the optometrist should ensure that the patient's eyeware provides adequate protection against ultraviolet rays. Evidence to date supports the use of a daily high-dose antioxidant supplement consisting of vitamins $\mathrm{C}$ and E, carotenoids, and zinc (i.e., the AREDS 2 formula), to reduce the risk of progression from intermediate to advanced AMD. Optometrists should recommend this type of supplement to patients with category 3 or 4 AMD to mitigate the risk of disease progression. The benefits in patients with milder forms of AMD or in those who are at risk of developing the disease is less clear. In such cases, it could be beneficial to recommend an omega-3 supplement, and eventually vitamin supplements, based on the patient's diet quality. The results of observational studies, including those derived from the original AREDS study, suggest that omega-3 supplementation may help to prevent or slow the progression of disease in its early stage. This approach has biological plausibility, since omega- 3 fatty acids are concentrated in the retina and have been shown to modulate retinal function. 
On the basis of the recently reported AREDS 2 study, substitution of beta-carotene by lutein plus zeaxanthin seems reasonable, since these carotenoids have been shown to protect against AMD without the associated risk of lung cancer in smokers. Consequently, any patient who has previously been a smoker or been exposed to second-hand smoke should consider taking supplements that do not contain beta-carotene.

This group concluded that the full body of literature must be taken into consideration in order to justify clinical recommendations for patients. A single study cannot, by itself, guide clinical practice. In all cases, recommendations must be individualized and patients should be monitored regularly.

\section{ACKNOWLEDGEMENTS}

The meeting of this regional group of experts was made possible by an education grant from Alcon Canada.

\section{REFERENCES}

1. Friedman DS, O'Colman BJ, Munoz B, et al. Prevalence of age-related macular degeneration in the United States. Arch Ophthalmol 2004;122:564-72.

2. Wong IYH, Koo SCY, Chan CWN. Prevention of age-related macular degeneration. Int Ophthalmol 2011;31:73-82.

3. Brown MM, Brown GC, Stein JD, et al. Age-related macular degeneration: economic burden and value-based medicine analysis. Can J Ophthalmol 2005;40:277-87.

4. Klein R, Klein BE, Linton KL. Prevalence of agerelated maculopathy: the Beaver Dam Eye Study. Ophthalmology 1992;99:933-43.

5. Klein R, Klein BE, Tomany SC, et al. Ten-year incidence and progression of age-related maculopathy: the Beaver Dam eye study. Ophthalmology 2002;109:1767-79.

6. Age-Related Eye Disease Study Research Group. A randomized, placebo-controlled, clinical trial of high-dose supplementation with vitamins $\mathrm{C}$ and $\mathrm{E}$, beta-carotene, and zinc for age-related macular degeneration and vision loss: AREDS Report No. 8. Arch Ophthalmol 2001;119:1417-36.

7. Moeller SM, Parekh N, Tinker L, et al. Associations between intermediate age-related macular degeneration and lutein and zeaxanthin in the Carotenoids in Age-Related Eye Disease Study (CAREDS): Ancillary study of the Women's Health Initiative. Arch Ophthalmol 2006;124:1151-62.

8. Ho L, van Leeuwen R, Witteman JCM, et al. Reducing the genetic risk of age-related macular degeneration with dietary antioxidants, zinc, and $Ð-3$ fatty acids. Arch Ophthalmol 2011;129:758-66.

9. Age-Related Eye Disease Study Research Group. The relationship of dietary lipid intake and age-related macular degeneration in a case-control study. AREDS Report No. 20. Arch Ophthalmol 2007;125:671-9.

10. SanGiovanni JP et al. Đ-3 long-chain polyunsaturated fatty acid intake and 12-y incidence of neovascular age-related macular degeneration and central geographic atrophy: AREDS report 30, a prospective cohort study from the Age-Related Eye Disease Study. Am J Clin Nutr 2009;90:1601-7.

11. Pareykh N, Voland RP, Moeller SM, et al. Association between dietary fat intake and age-related macular degeneration in the Carotenoids in Age-Related Eye Disease Study (CAREDS): An ancillary study of the Women's Health Initiative. Arch Ophthalmol 2009;127:1483-93.

12. Christen WG, Schaumberg DA, Glynn RJ, et al. Dietary Đ-3 fatty acid and fish intake and incident age-related macular degeneration in women. Arch Ophthalmol 2011;129:921-9.

13. van Leeuwen $\mathrm{R},<\mathrm{AU}$ : Please provide at least two more author names >et al. Dietary intake of antioxidants and risk of age-related macular degeneration. JAMA 2005;294:2101-7.

14. The Age-Related Eye Disease Study 2 (AREDS2) Research Group. Lutein + zeaxanthin and omega-3 fatty acids for age-related macular degeneration: The Age-Related Eye Disease Study 2 (AREDS2) randomized clinical trial. JAMA 2013;309:doi:10.1001/ jama.2013.4997.

15. Acs M, Kaplan M, Barrie D. The Macular Assessment Program. Abstract presented at the American Academy of Optometry Annual Meeting, 23-26 October 2013, Seattle (Abstract 130959).

16. Druesne-Pecollo N, Latino-Martel P, Norat T, et al. Beta-carotene supplementation and cancer risk : a systematic review and meta-analysis of randomized controlled trials. Int J Cancer 2010;127:172-84.

17. Health Canada. Canadian Tobacco Use Monitoring Survey, February-December 2012. www.hc-sc.gc.ca hc-ps/tobac-tabac/research-recherche/stat/_ctumsesutc_2012/ann-eng.php. Accessed November 5, 2013.

18. Cho E, Hung S, Willett WC, et al. Prospective study of dietary fat and the risk of age-related macular degeneration. Am J Clin Nutr 2001;73:209-18. 


\section{CTOPCON.}

CONNECTING VISIONS
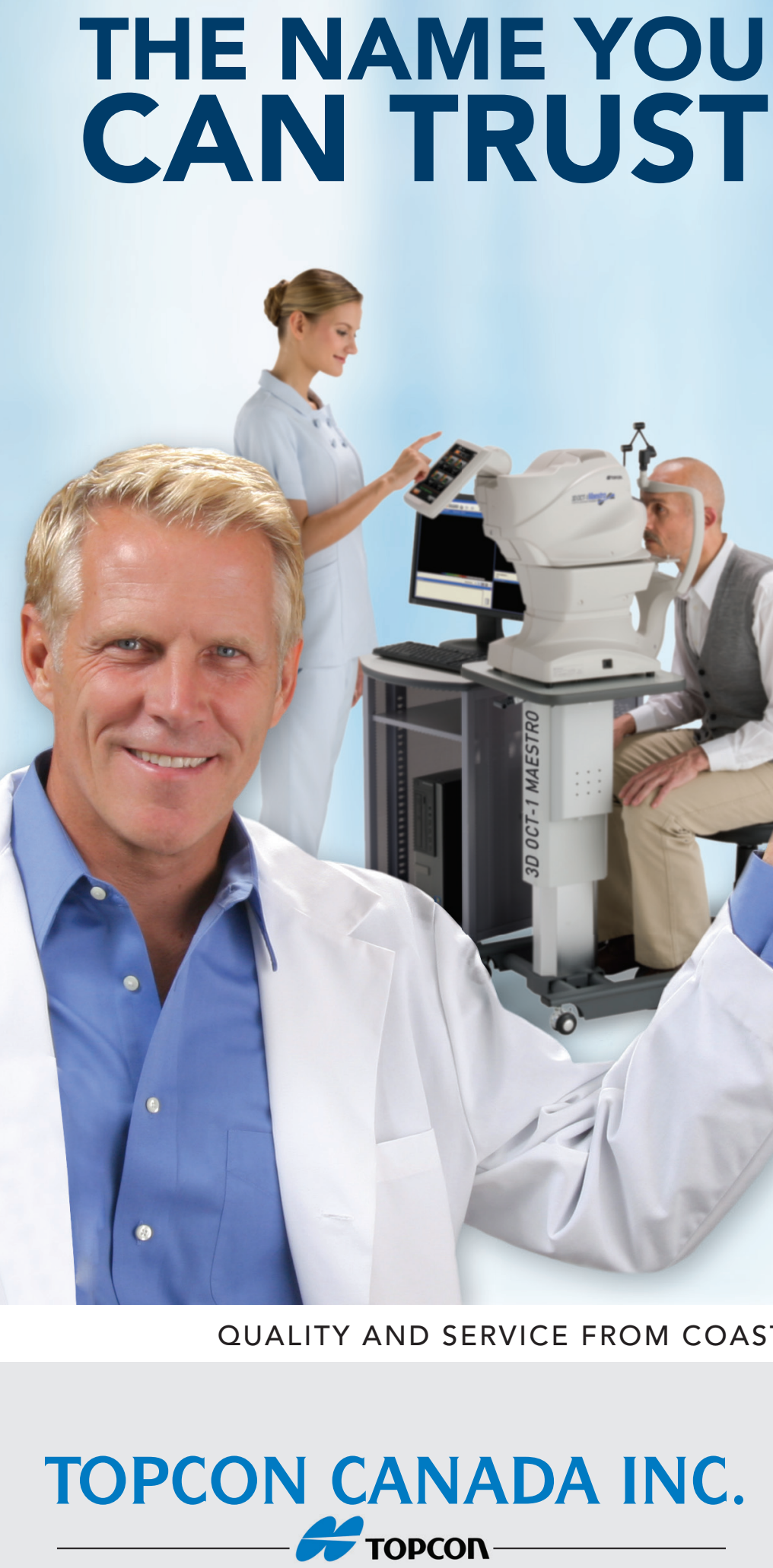

Exclusive Canadian distributor for:

Topcon, Amtek, Welch Allyn, Gulden, M\&S Technologies, Icare, Mortan
- Exciting new products

- Leading technologies

- Unparalleled support

- Superior quality at competitive pricing
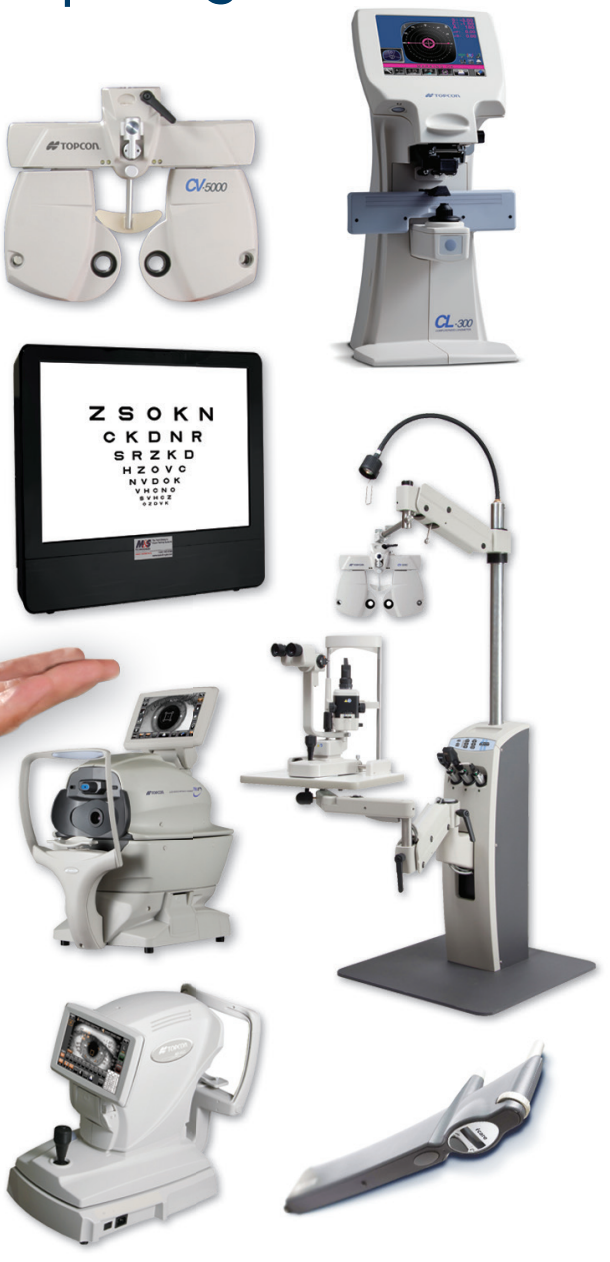


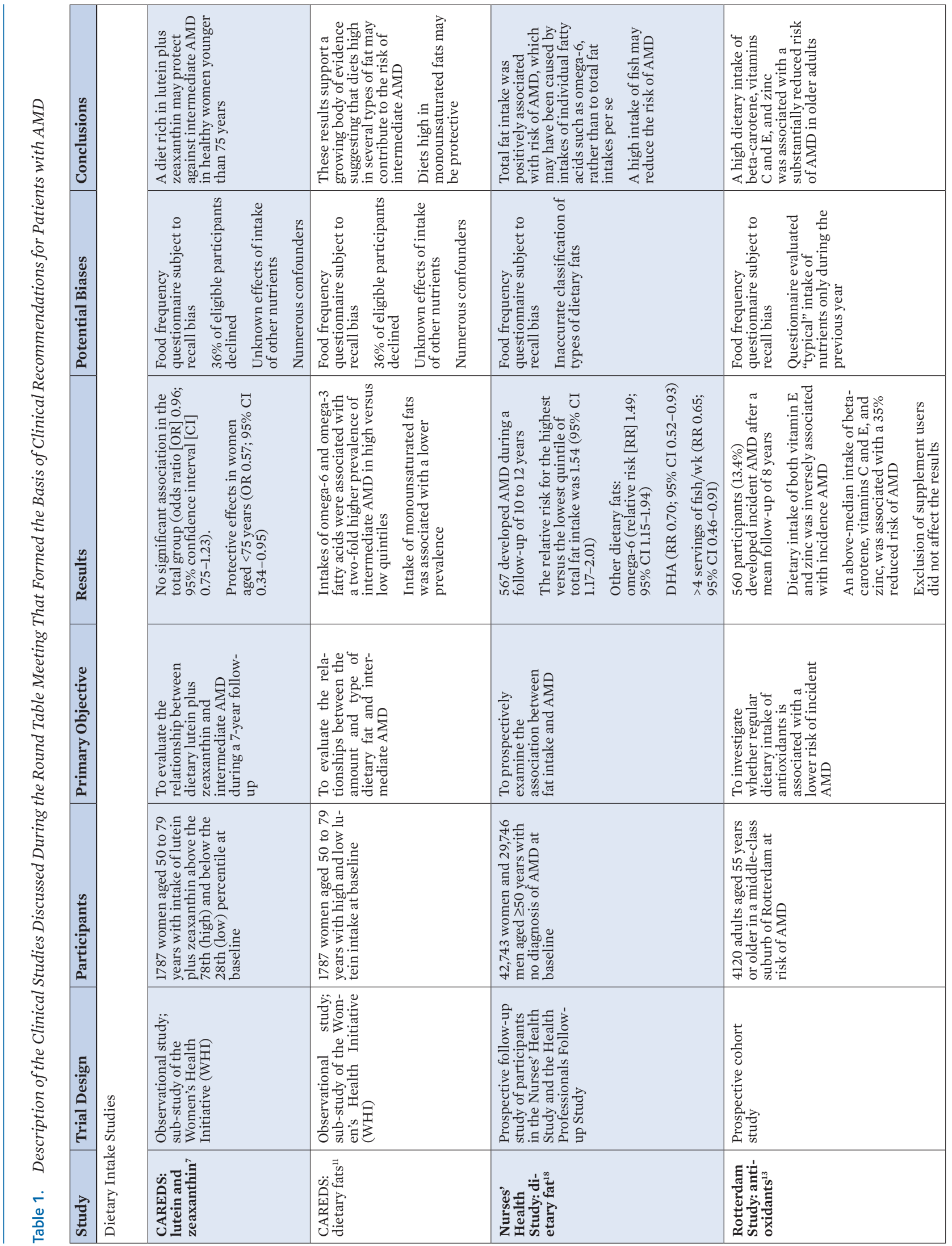




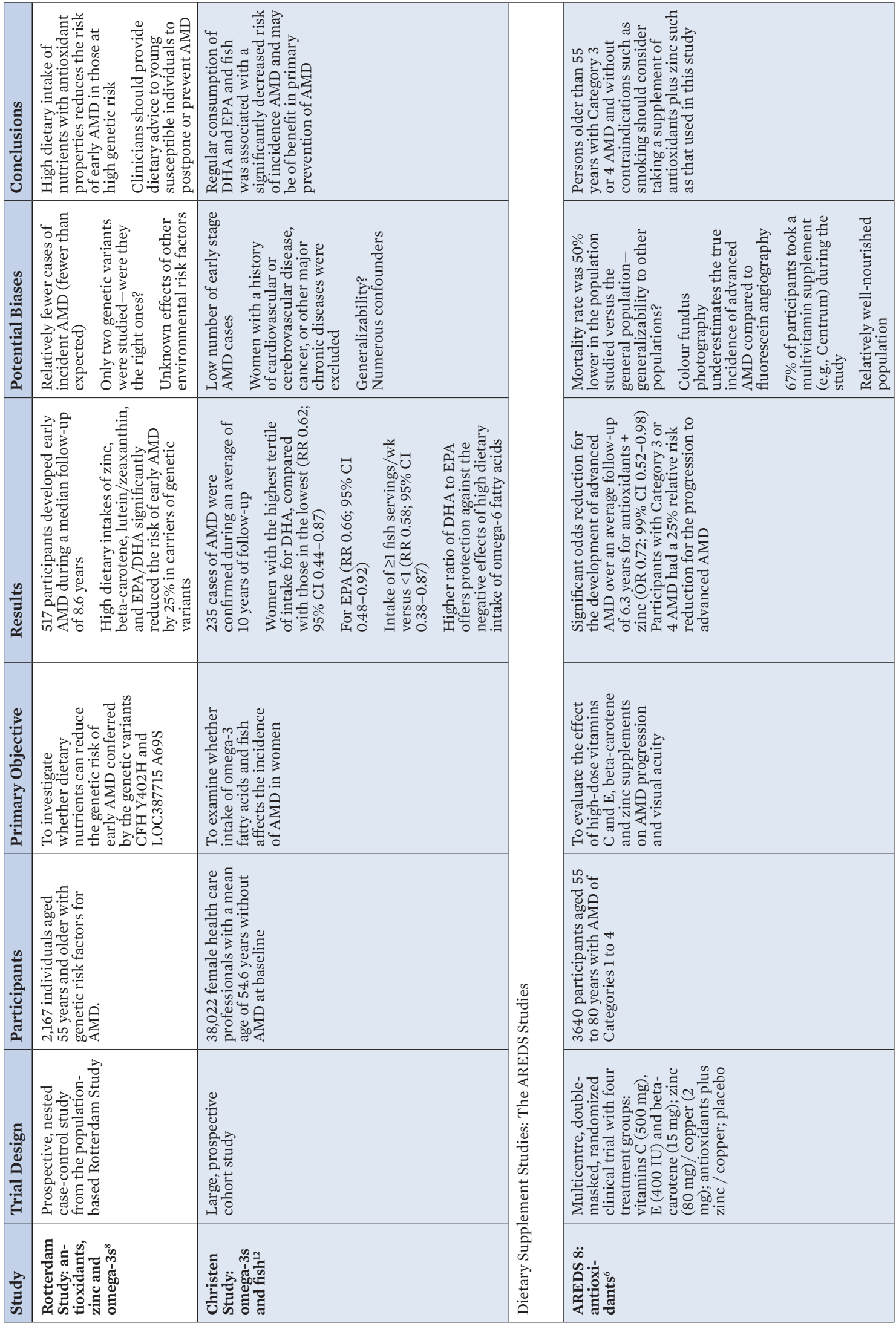




\begin{tabular}{|c|c|c|c|}
\hline 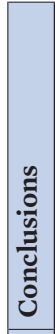 & 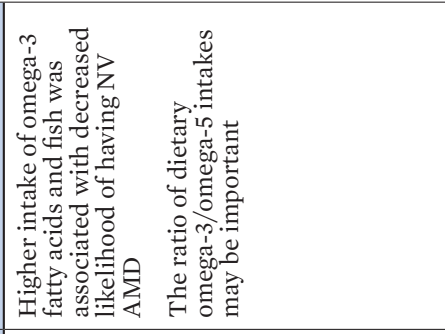 & 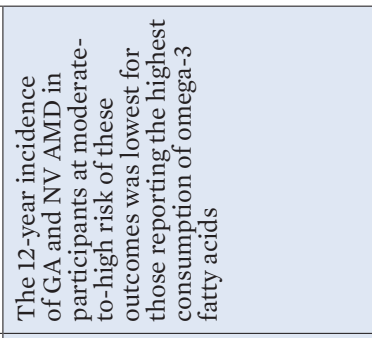 & 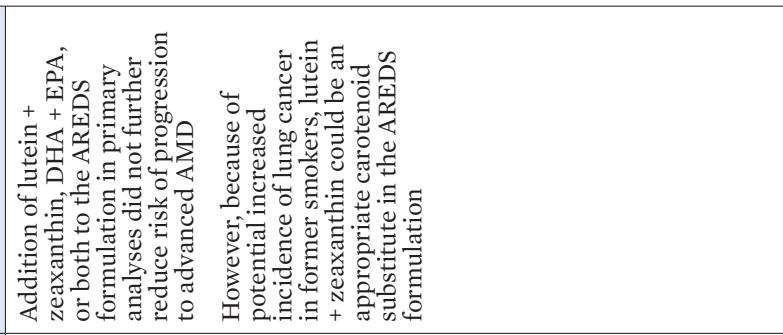 \\
\hline 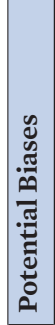 & 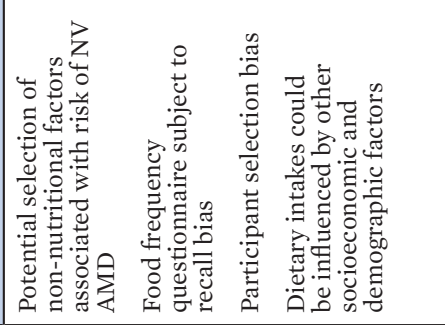 & 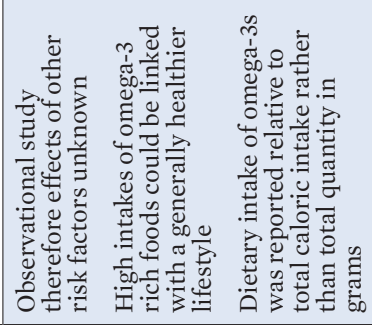 & 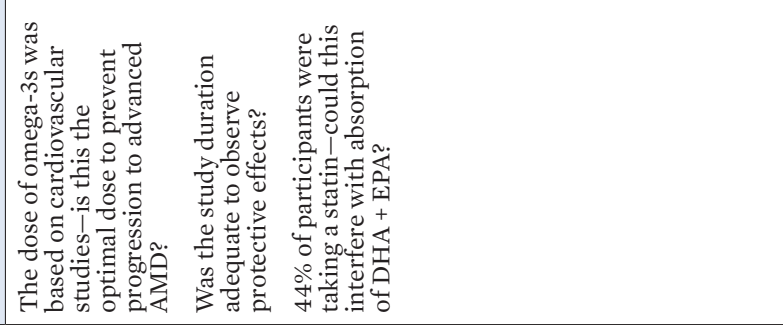 \\
\hline 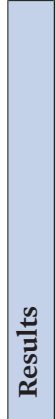 & 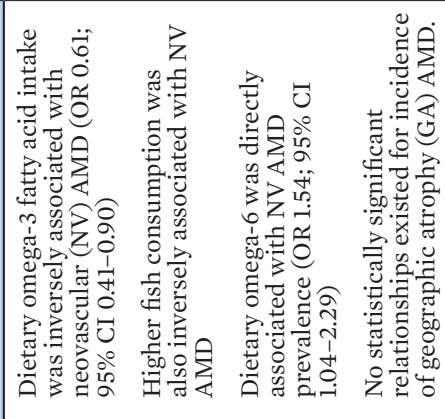 & 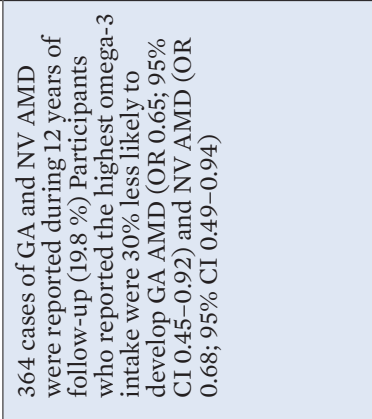 & 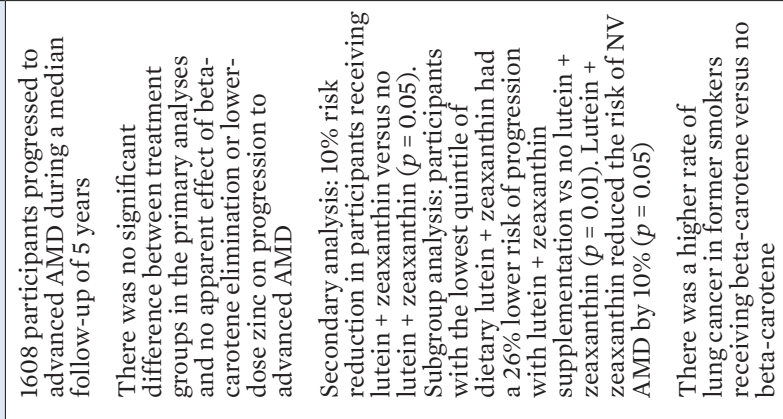 \\
\hline 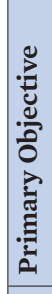 & 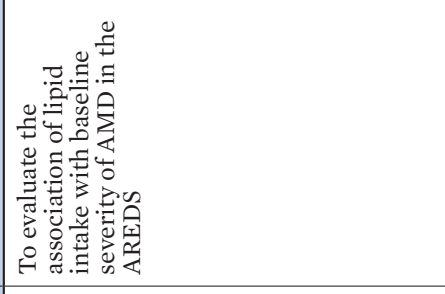 & 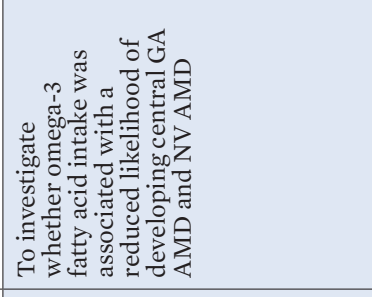 & 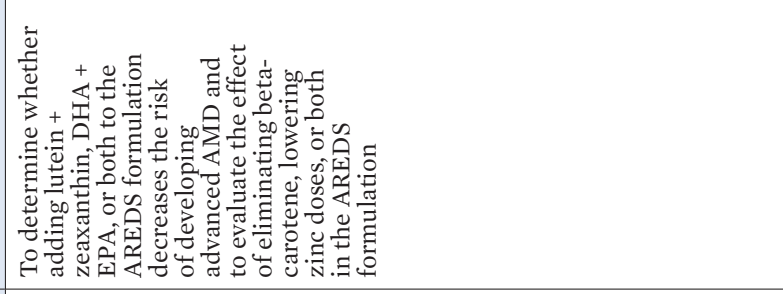 \\
\hline 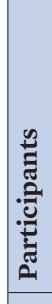 & 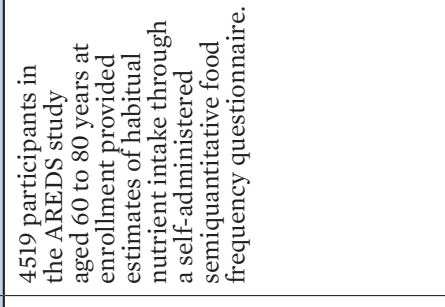 & 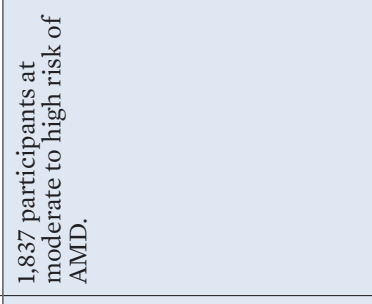 & 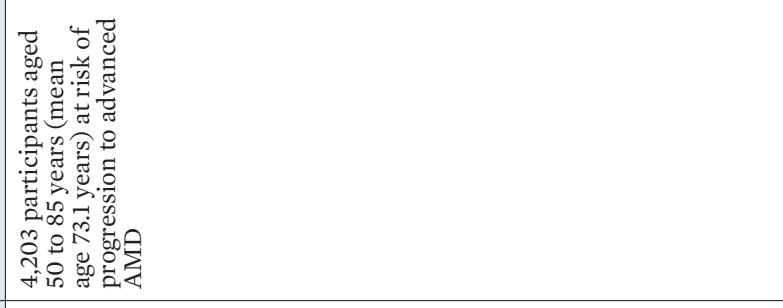 \\
\hline 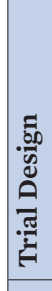 & 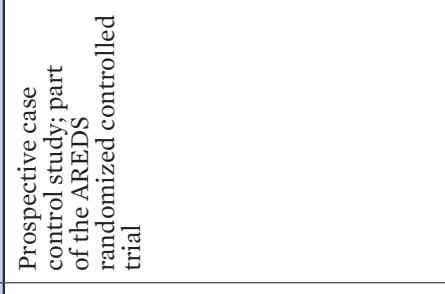 & 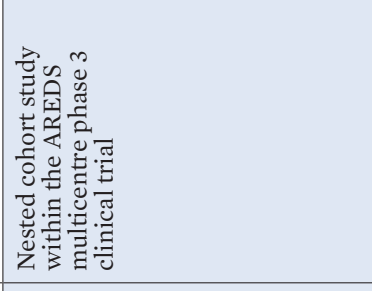 & 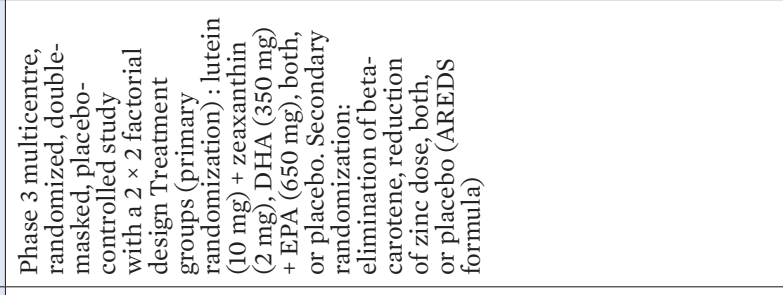 \\
\hline E & 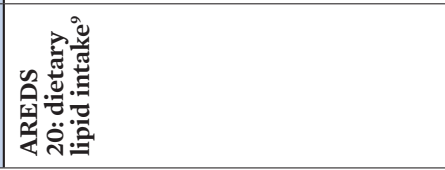 & 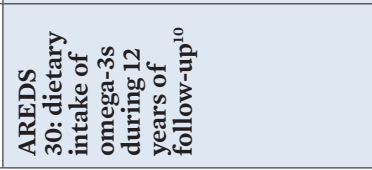 & 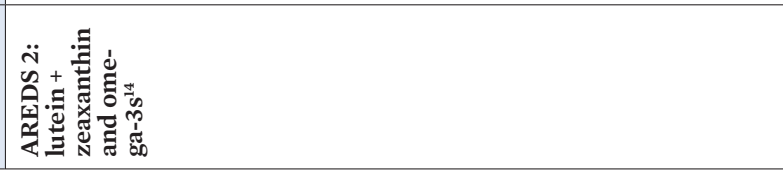 \\
\hline
\end{tabular}

